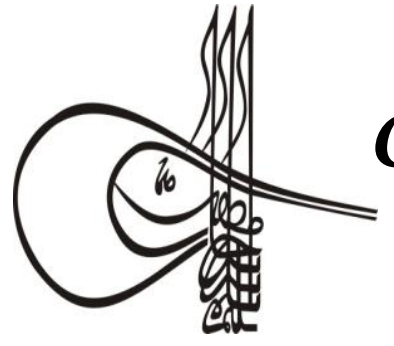

Comparative Religious Studies

Volume 14 Issue 2, 2019, p. 237-249

DOI: 10.29228/TurkishStudies.22690

ISSN: 2667-5544

Skopje/MACEDONIA-Ankara/TURKEY

Research Article / Araştırma Makalesi

Article Info/Makale Bilgisi

Received/Geliş: 10.01 .2019

Gor Report Dates/Rapor Tarihleri: Referee 1 (12.02.2019)- Referee 2 (14.03.2019)

This article was checked by intihal.net.
2

INTERNATIONAL

BALKAN

UNIVERSITY

EXCELLENCE FOR THE FUTURE IBU.EDU.MK

\title{
من مبادئ العلاقات الدولية في القرآن الكريم
}

\author{
Mesut CEVHER*
}

جاء القرآن الكريم في وقت كان العدوان فيه علامة على القوة، فكانت الدولة القوية تغزو الدول



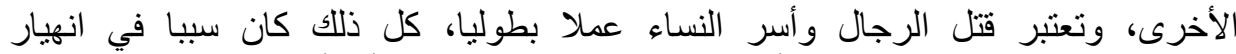

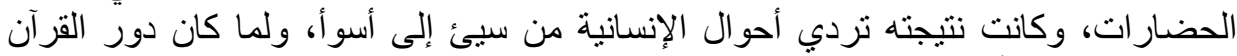



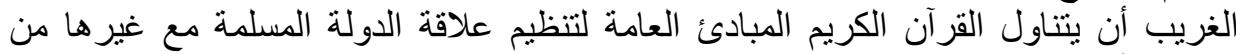



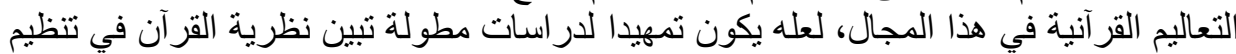

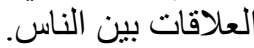

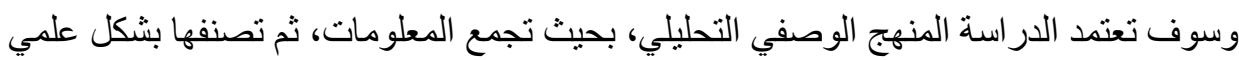



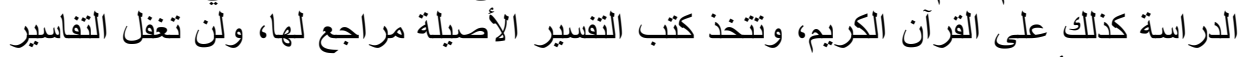
و الدر اسات الأكاديمية الحديثة. تمهيد

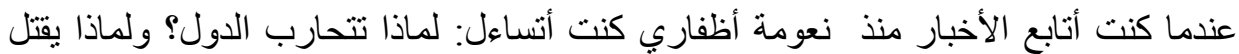

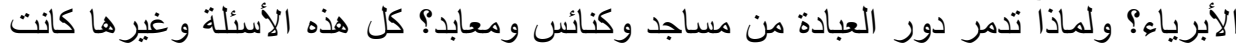



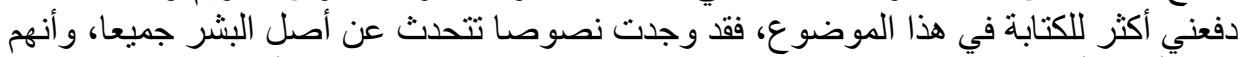

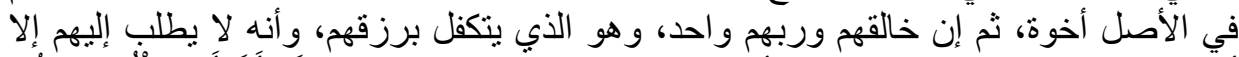

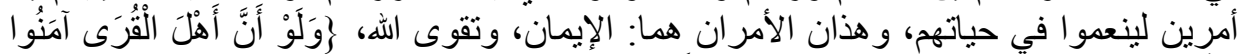

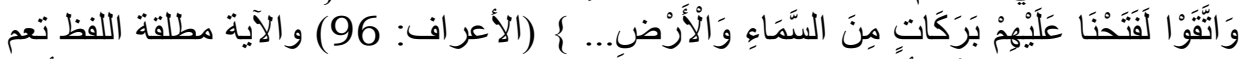

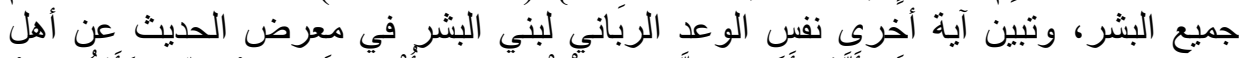






يكون الرزق ميسرا، وهكذا يكون متنوعا، فيأتي بسهولة من السماء، ويخرج دون عناء من



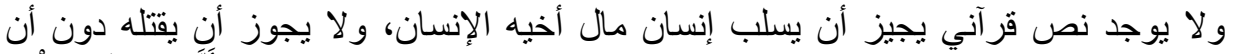



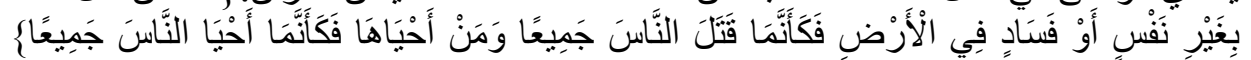

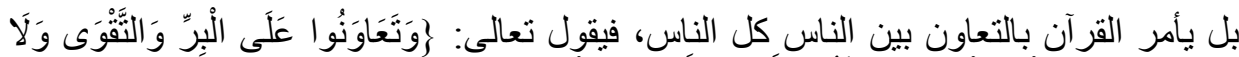

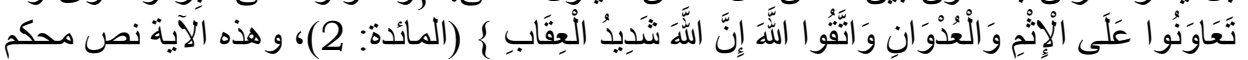

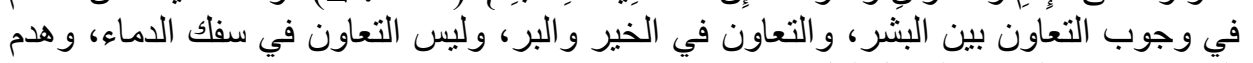
الحضار ات، والتدمير الثنامل لكل شيء.



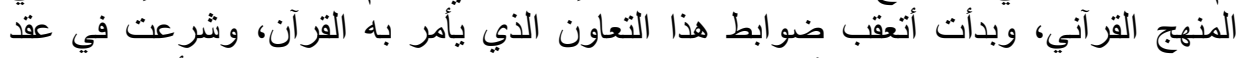

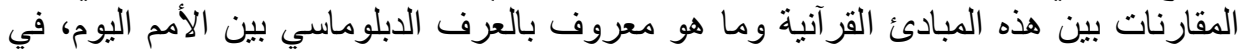



$$
\text { الهدف من البحث وأهميته: }
$$

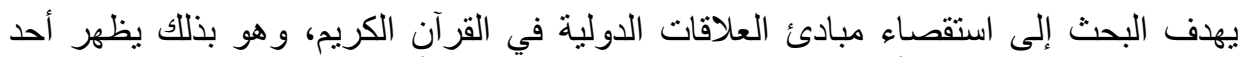

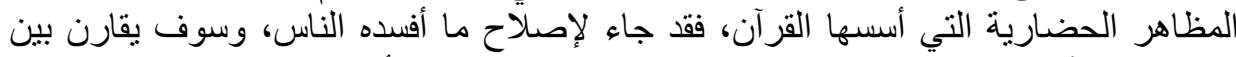

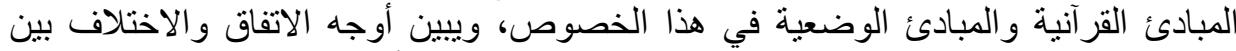

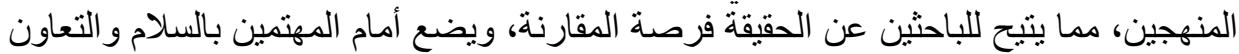



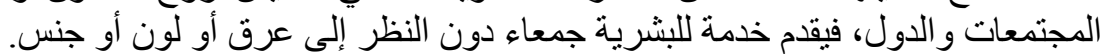

$$
\begin{aligned}
& \text { خطة البحث: }
\end{aligned}
$$



$$
\begin{aligned}
& \text { التمهيد } \\
& \text { المبحث الأول: تحريم العدوان: } \\
& \text { المبحث الثاني: المعاملة بالمثل: } \\
& \text { المبحث الثالث: وجوب الوفاء بالالتزامات و المعاهدات: } \\
& \text { المبحث الرابع: إعلام الخصوم قبل فسخ العقود: } \\
& \text { المبحث الخامس:حق اللجوء مكفول لجميع البشر: } \\
& \text { ثبت المصادر و المراجع } \\
& \text { الكلمات المفتاحية: العلاقات الدوليةـ العهدــ القرآن الكريم ـ المعاهدات. }
\end{aligned}
$$

\title{
SOME PRINCIPLES OF INTERNATIONAL RELATIONS IN THE HOLY QURĀN
}

\begin{abstract}
The Qur'an came at a time when aggression was a sign of strength. The powerful state was invading small countries, plowing and
\end{abstract}


plowing. The tribes were also proud of their attacks on other tribes. The killing of men and women's families is a heroic act. All of this was the cause of the collapse of civilizations, and the result of the deterioration of the conditions of humanity from bad to worse. And because the role of the Holy Qurān to reform what has been corrupted by human beings, and guide all mankind to the ways of happiness and nail. It is not surprising that the Holy Qurān deals with the general principles governing the relationship of the Muslim state with other countries. And to chart the way to a life where all human beings enjoy peace. The study will attempt to devise Qurānic teachings in this field, perhaps as a prelude to lengthy studies showing the theory of the Qurān in organizing relations between people.

The study will rely on the analytical descriptive method, so that the information is collected and classified scientifically according to the researcher, and then analyzed, in order to arrive at the facts scientifically. And will also study the Koran, and take the books of interpretation and authentic references. And will not ignore the interpretations and modern academic studies. This study will serve as a new door for researchers in the field of the Holy Qurān and its sciences, because it touched on unprecedented subject, needs to study and scrutiny, because it serves a vital area in the life of all mankind, and not just a religious study of those who convert to Islam.

\section{STRUCTURED ABSTRACT}

\section{Introduction}

When I was following the news from a very early age I was wondering: Why are countries fighting? Why kill innocent people? Why do houses of worship as mosques, churches and temples be destroyed? All these and other questions were motivated to search for a solution to many of what we suffer in this life. The more I read the Holy Quran, The more I want to write in this subject. I found texts that talk about the origin of all human beings, that they are originally brothers, and that their Creator and their Lord are one, He is the one who takes care of their sustenance, and that only two things are required for them to enjoy their lives. These are: faith, " And if only the people of the cities had believed and feared Allah, We would have opened upon them blessings from the heaven and the earth..." (Al-A'raf: 96) And another verse shows the same promise of the Lord of mankind in the context of the talk about the people of the book, says God

" And if only they upheld [the law of] the Torah, the Gospel, and what has been revealed to them from their Lord, they would have consumed [provision] from above them and from beneath their feet. Among them are a moderate community... "(Al-Maida: 66) Heaven, and come out without trouble from the ground.

There is no Qur'anic text that permits a person to take away the money of His brother in humanity

, and it is not permissible to kill him without assaulting him. Even if he is attacked, there is no need for a fair trial, so the Qur'an declares: " ...We decreed upon the Children of Israel that whoever kills a soul 
unless for a soul or for corruption [done] in the land - it is as if he had slain mankind entirely. And whoever saves one - it is as if he had saved mankind entirely... "(Al-Maida: 32)

But the Qurān commands the cooperation between people and all people, and the Almighty says: ( ...And cooperate in righteousness and piety, but do not cooperate in sin and aggression. And fear Allah; indeed, Allah is severe in penalty.) (Al-Maida: 2), and this verse text is tightly in the need to cooperate between human beings, Not cooperation in bloodshed, the destruction of civilizations, and the total destruction of everything.

I started looking for the framework that governs these human relations in the Qurānic approach. I began to follow the controls of this cooperation, which is ordered by the Qur'an. I began to compare these Qur'anic principles and what is known to the diplomatic tradition among the nations today in an attempt to understand what Characterized by the Koranic approach, and what we can offer in this framework to serve humanity.

Objective and importance of research: The research aims to investigate the principles of international relations in the Holy Quran, thus showing one of the cultural aspects established by the Qur'an. It came to reform what people have corrupted. It will compare Qurānic principles and positivist principles in this regard. The truth is the opportunity of comparison, and it sets before those interested in peace and cooperation a plan to cure our world of conflicts, and helps to establish a spirit of cooperation and understanding between societies and nations, serving all humanity without regard to race, color or sex.

Search Plan:

Search section on: Introduction:

The first topic: Prohibition of aggression:

The second topic: Reciprocity:

The third topic: The obligations and treaties must be fulfilled:

The fourth topic: informing the litigants before the dissolution of contracts:

The fifth topic: The right of asylum is guaranteed to all human beings: sources and references

Keywords: International Relations - The Covenant - The Holy Qurān - Treaties.

تعتبر العلاقات الدولية شريان حياة الدول، وهي في كثير من الأحيان تيسر حياة المواطنين، أو تعقدها وتقيد الطيد

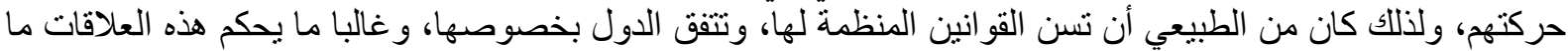

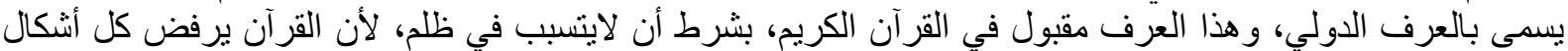

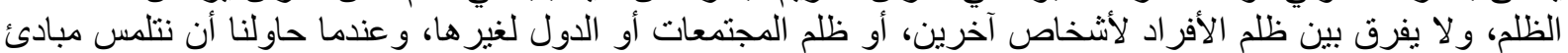




1 المبحث الأول:تحريم العدوان:

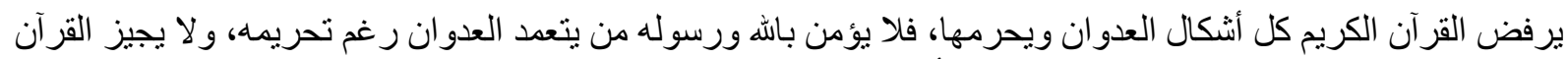

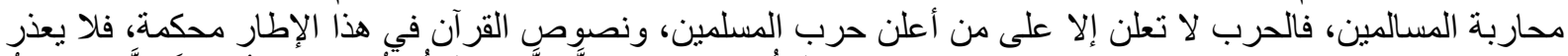

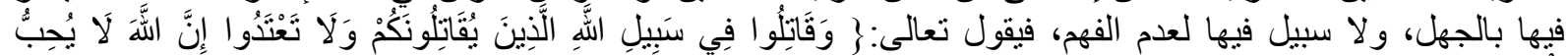

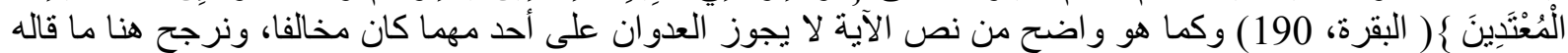



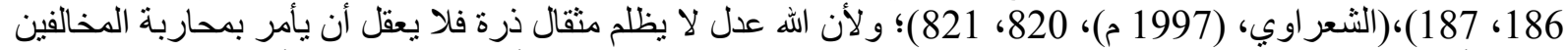



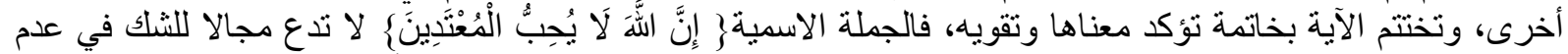



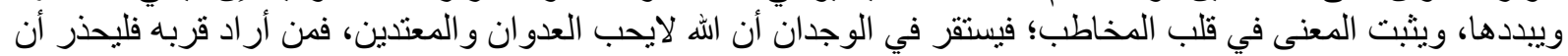

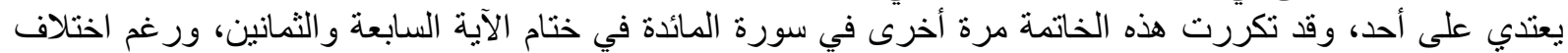

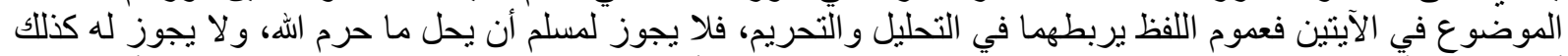

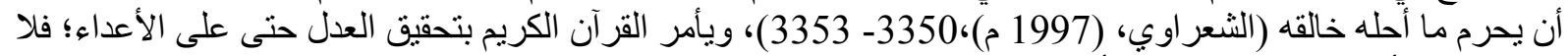

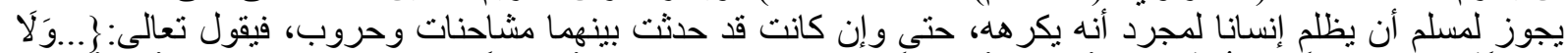

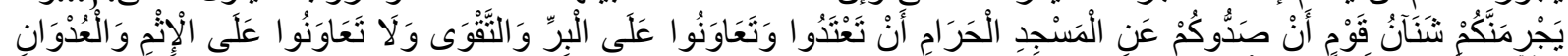



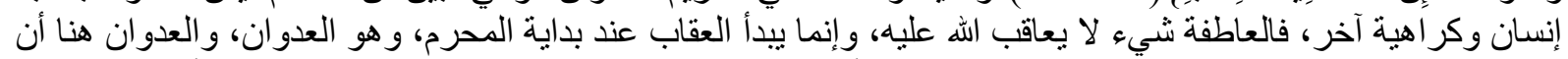



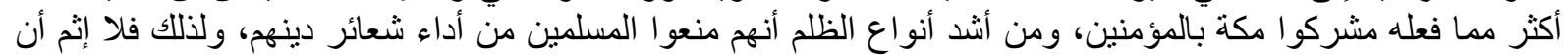

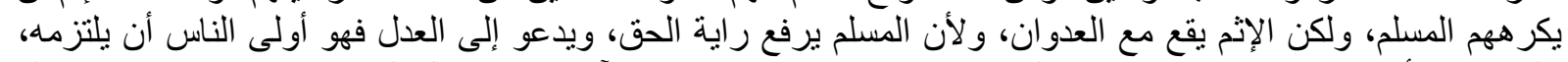



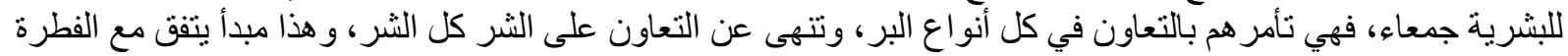



(2912 - 2897، (1997)

\section{2}

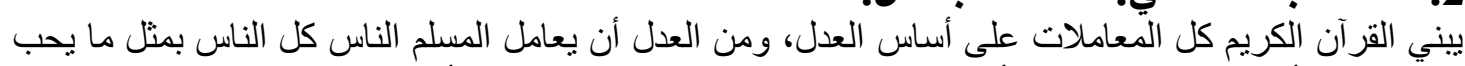

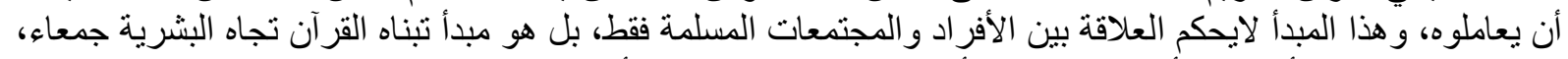

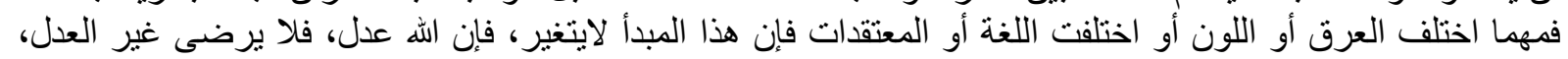

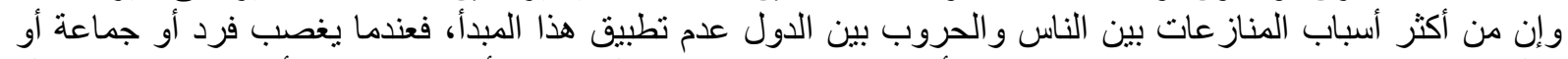

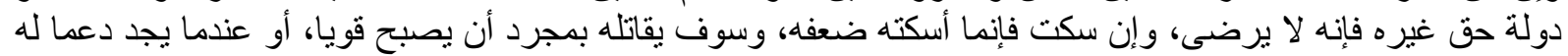

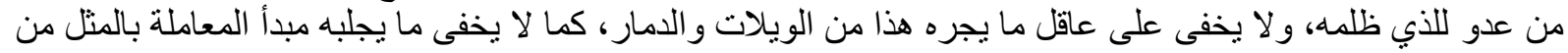
رضا ورخاء وتقدم للبشرية.

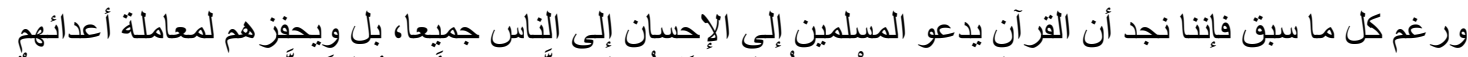

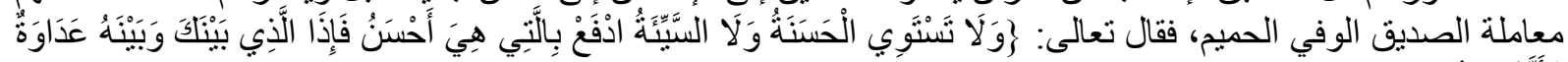

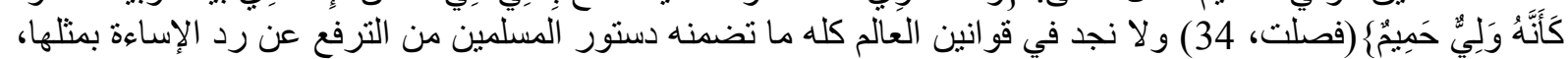

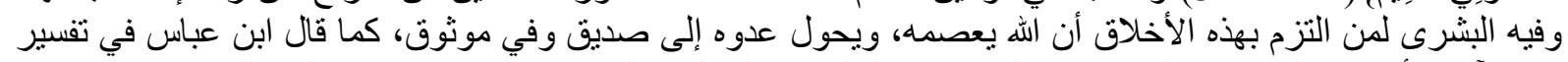

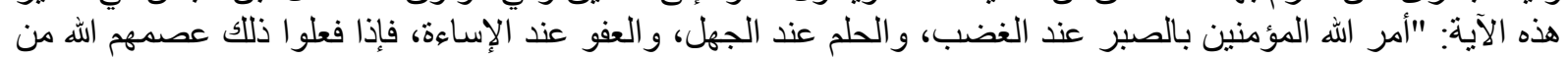

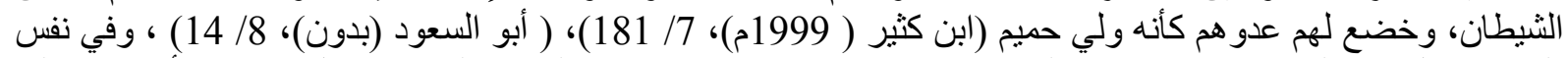



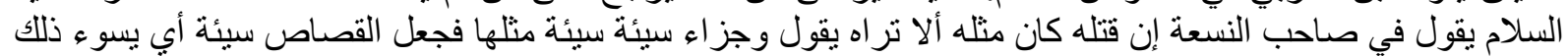
الفعل مع كونه مشرو عا فمن عفا وأصلح فأجره على الله." (ابن العربي، (1946)، 167,168) وتعاليخ الإنسلام تُؤكد أن اليد 


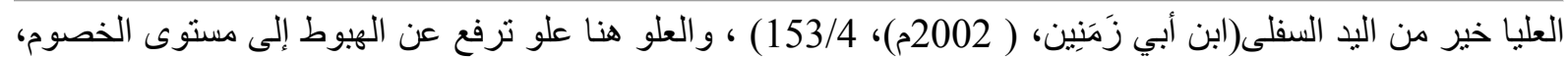





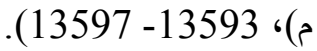

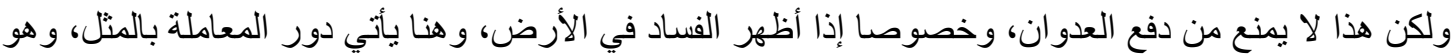

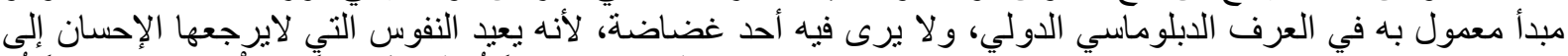

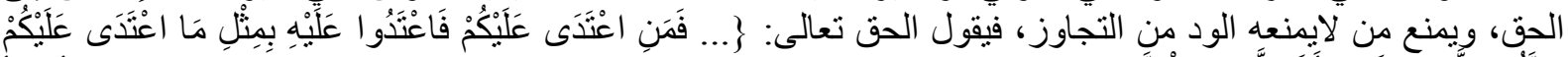

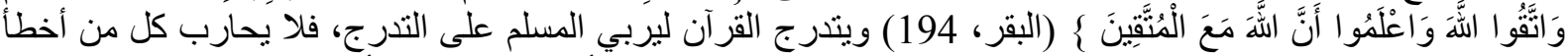



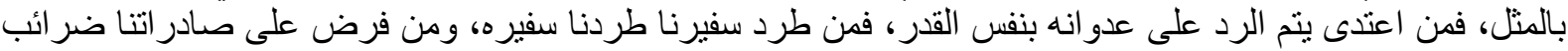

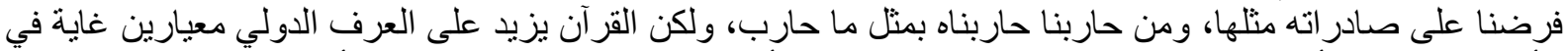

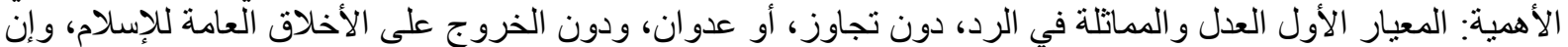



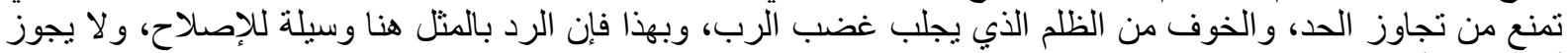



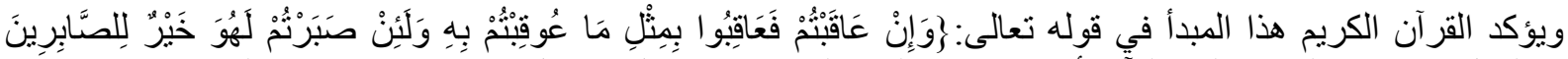





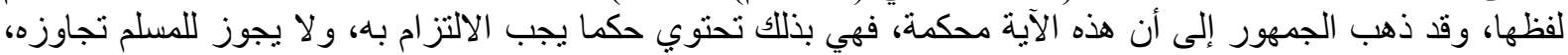
و هو أدعى إلى إنشاء علاقات قوية بين بني البشر، يكون الأساس فيها أن الناس سواسية كأسنان المشط (ابن لطف الله

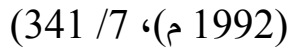



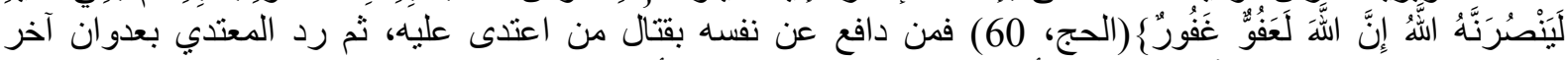

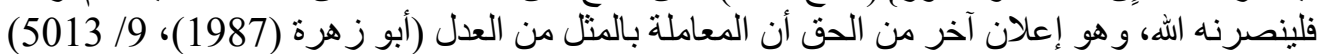

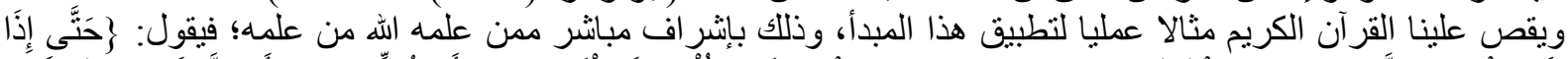

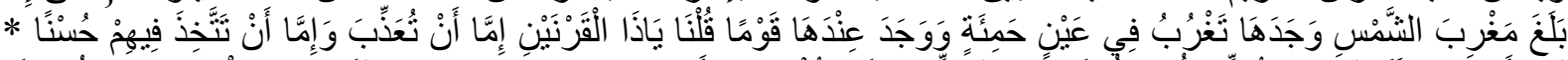

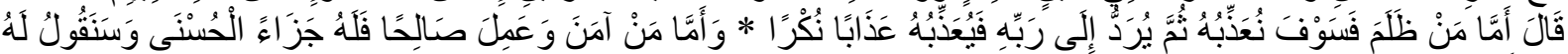

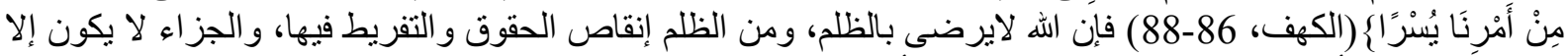

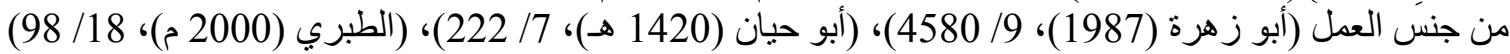

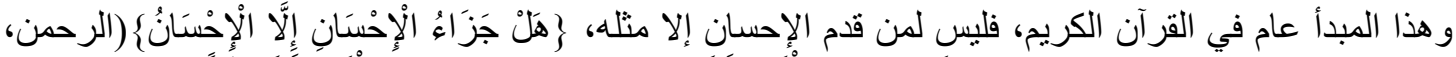



\section{3. المبحث الثالث: وجوب الوفاء بالالتزامات والمعاهدات:}

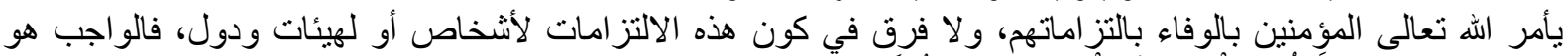

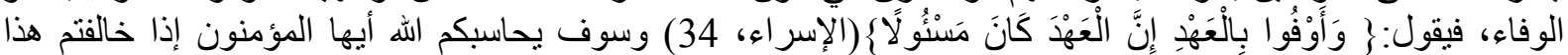



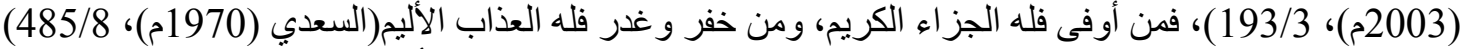

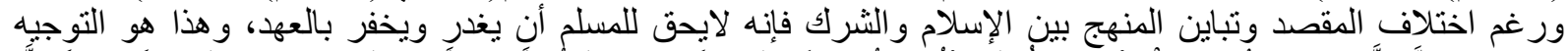

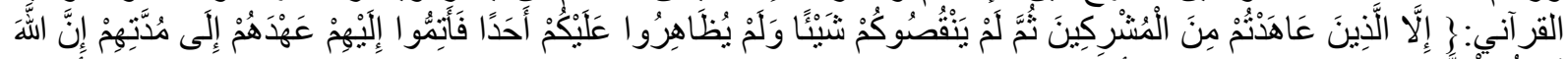

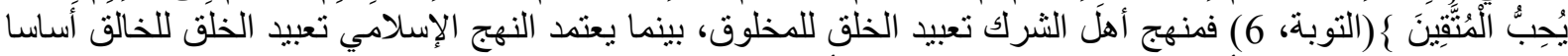

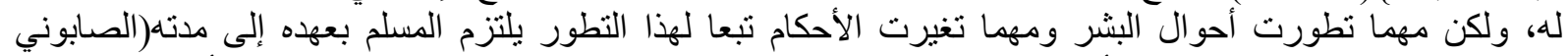

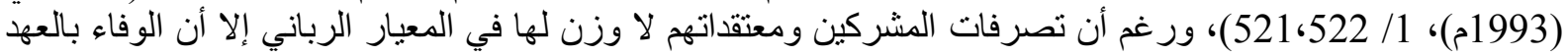

\section{Turkish Studies - Comparative Religious Studies}

Volume 14 Issue 2, 2019 






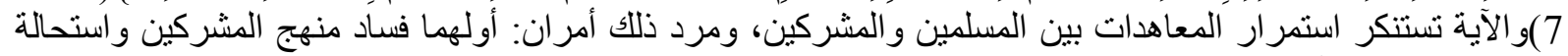

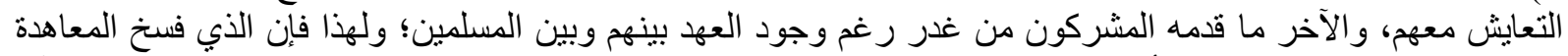

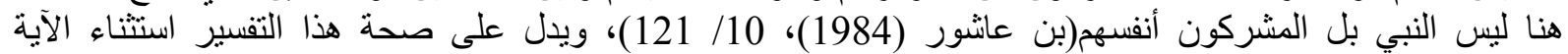

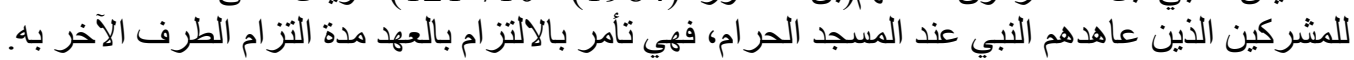

\section{4. المبحث الرابع:إعلام الخصوم قبل فسخ العقود:}

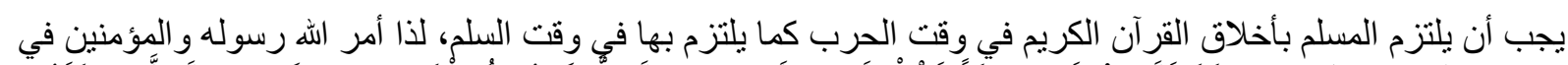

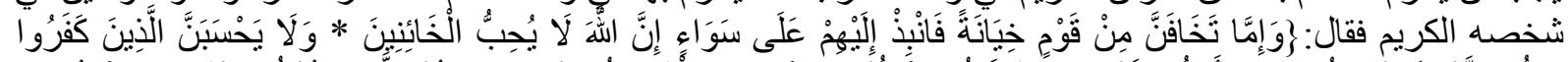



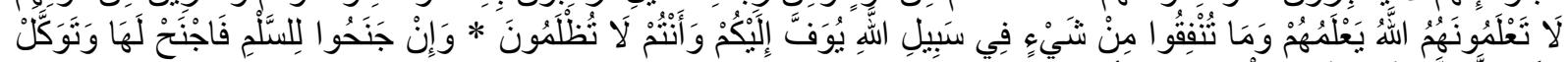

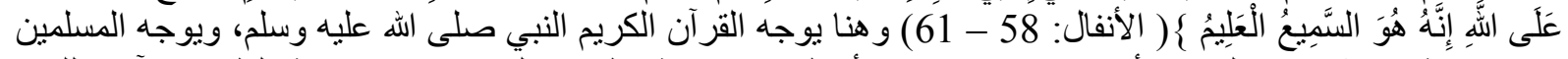

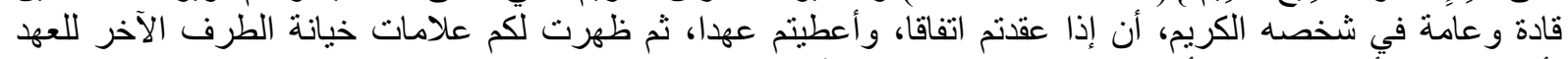



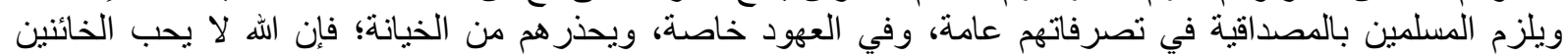

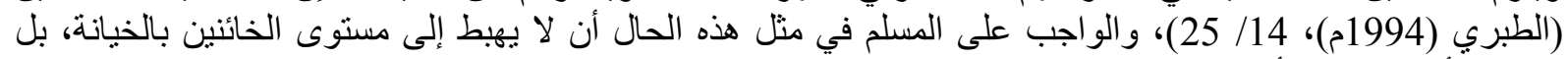

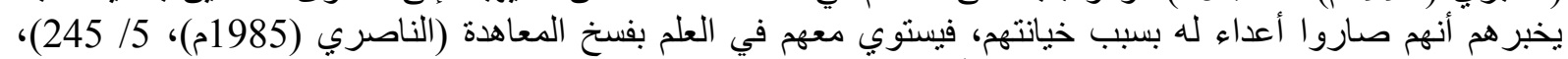

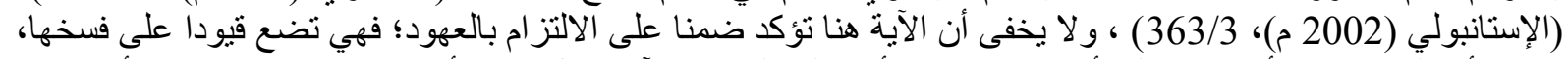

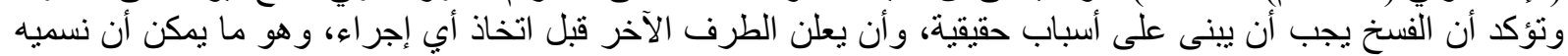

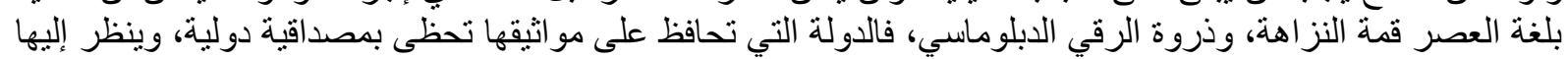

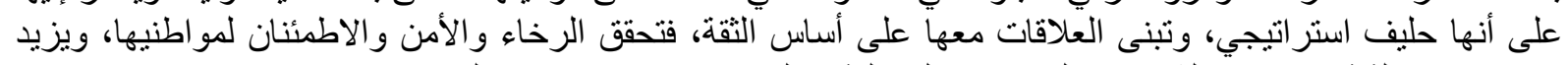

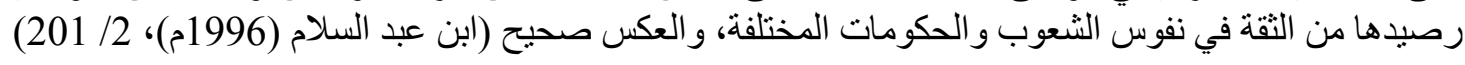

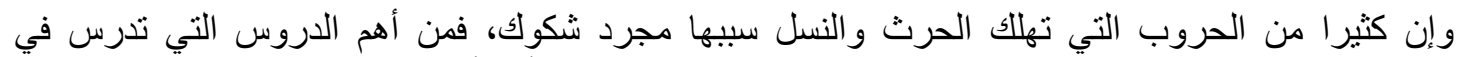

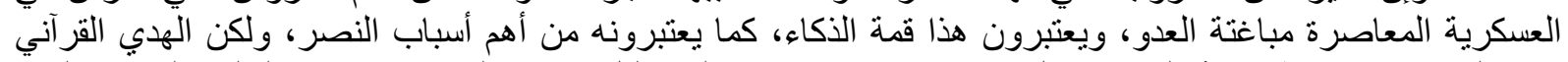

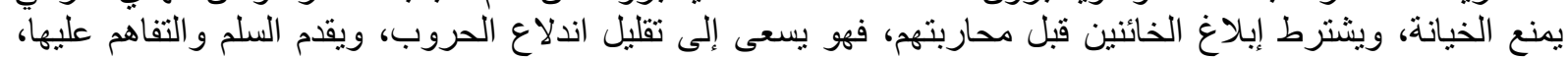





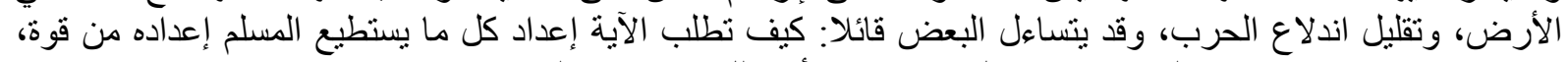



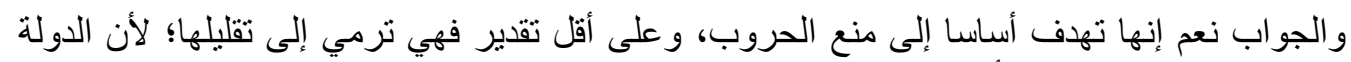

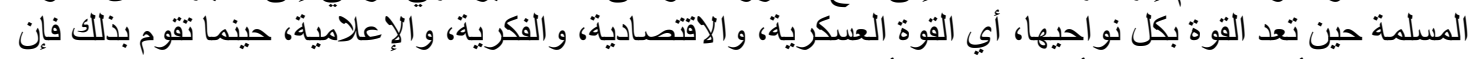

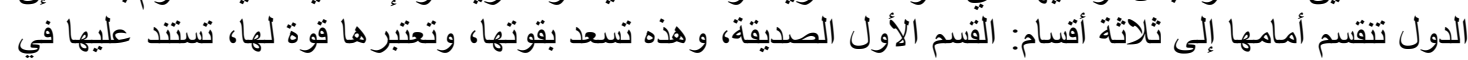

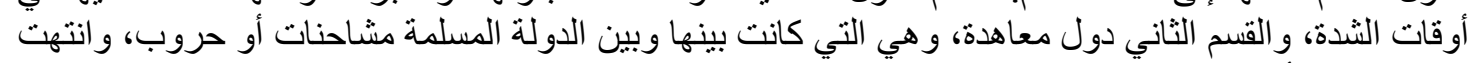

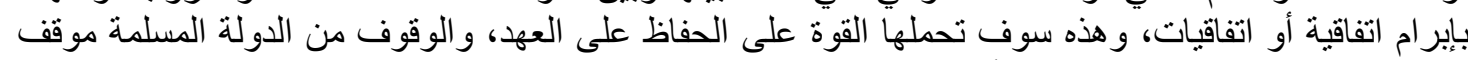

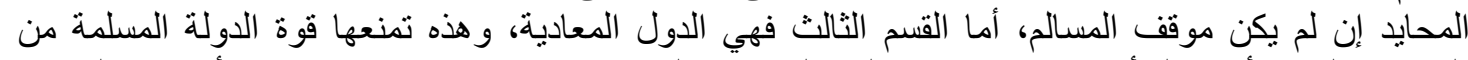





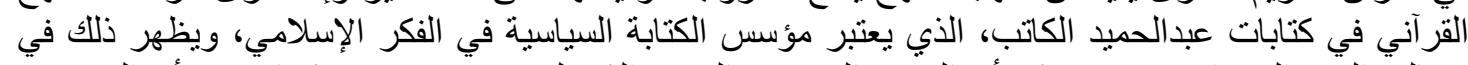





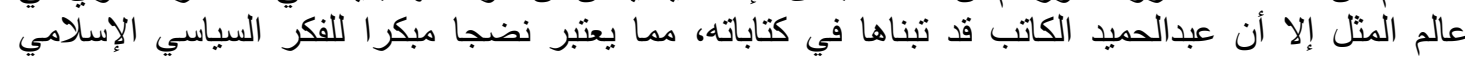


و وآيات القرآن في هذا الصدد كثثرة، و لا (Gündüzöz,Soner- Gündüzöz,Güldane. (2007), 73, 74.)

يتسع المجال لذكر ها هنا، فلعلنا نقوم بذللك في دراسة تفصيلية لاحقة.

\section{5. المبحث الخامس:حق اللجوء مكفول لجميع البشر:}

يقدم القر آن الكريم نموذجا لحماية اللاجئين لا يدانيه نموذج، فقبل أكثر من ألف وأربعمائة سنة، وقبل أنيل أن يسمع أحد



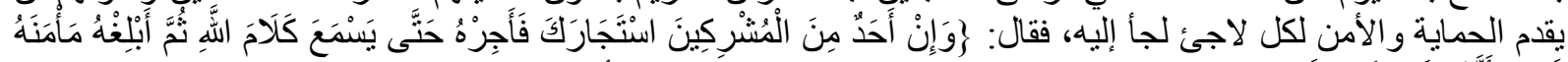

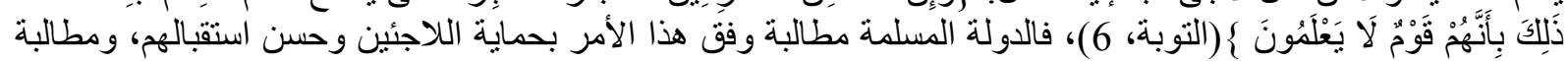









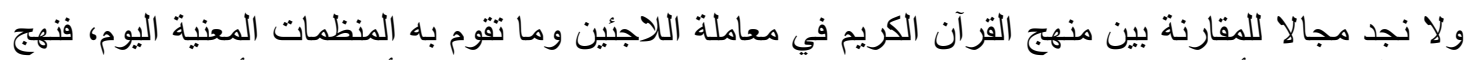

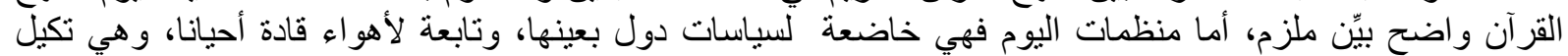

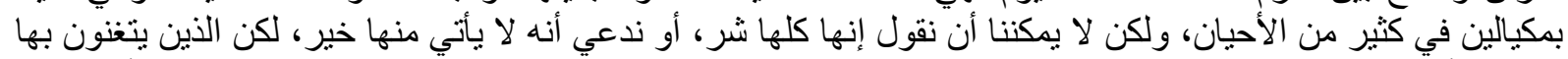

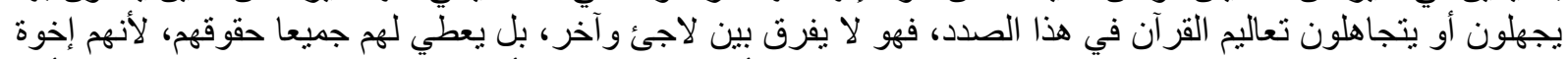

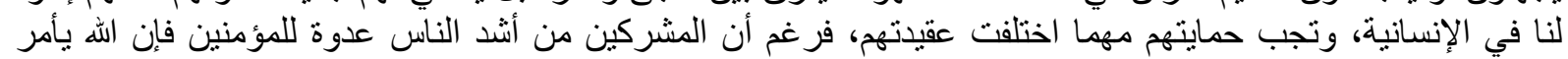



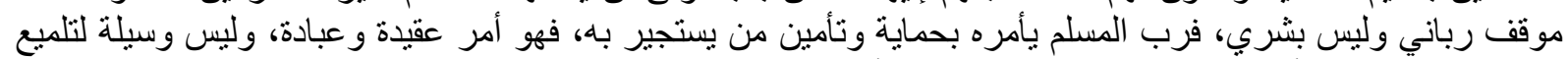

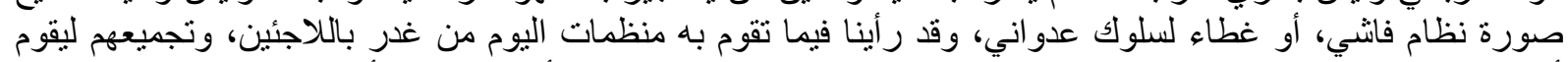

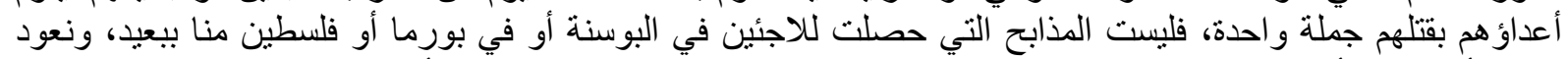







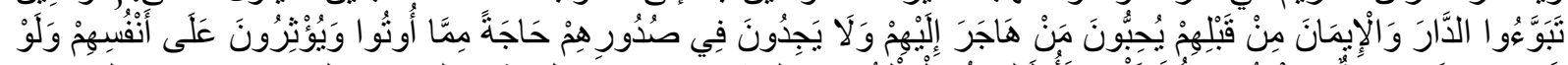



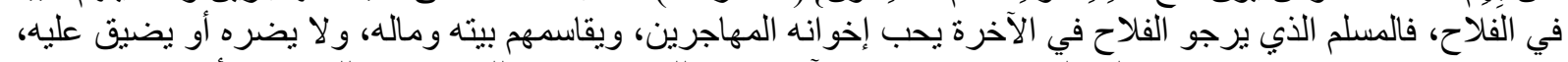

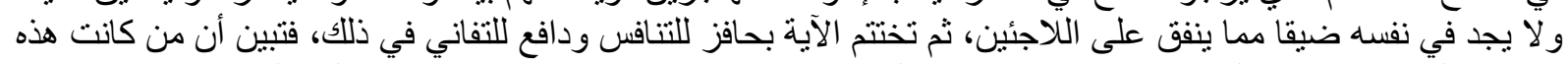

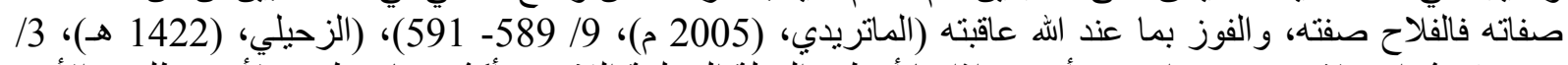

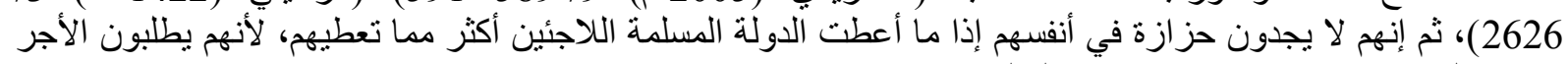







وقد يقول قائل إن هذا يوجه إليه القرآن تجاه المهاجرين من المسلمين، والحقيقة أن المنهج القرآني لا يفرق بين الئن المسلم

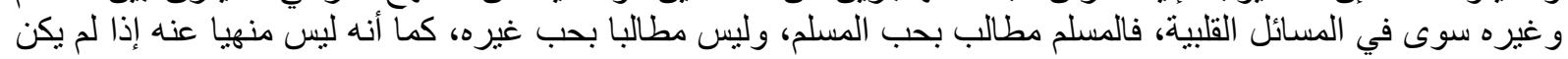







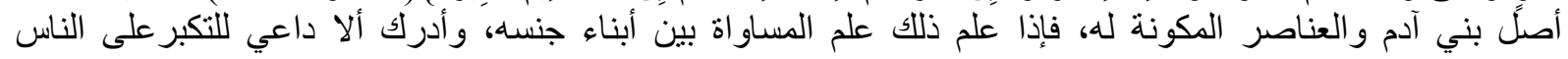




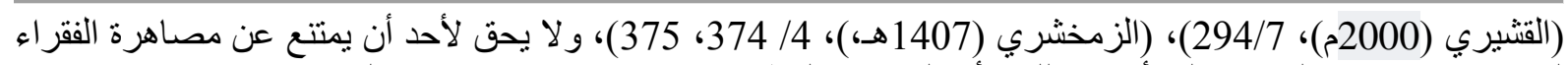



\section{الخاتمة}

يبين القرآن الكريم أن الناس جميعا في الأصل أمة واحدة، وأن سبب التفرق الذي وقع بين الناس هو اختاف الألاف

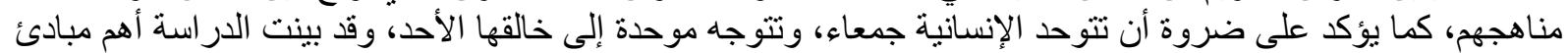

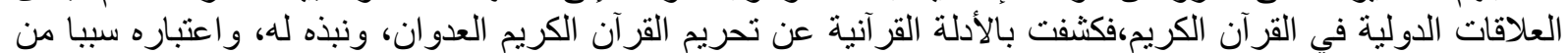





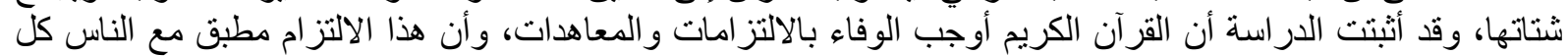

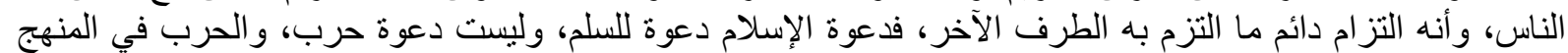



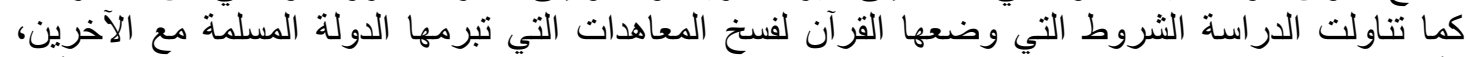

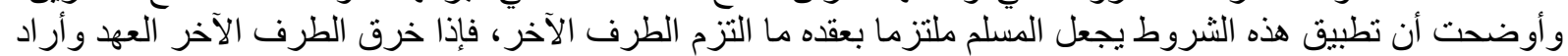

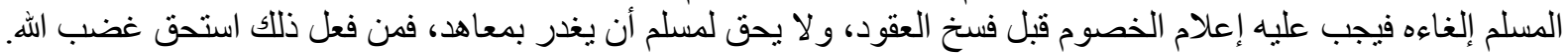

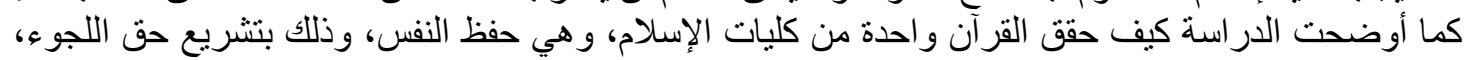

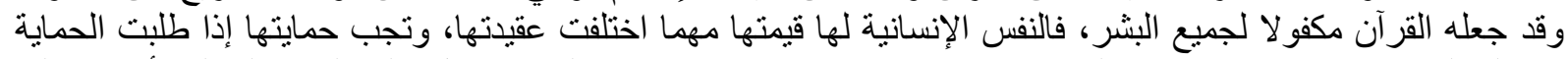

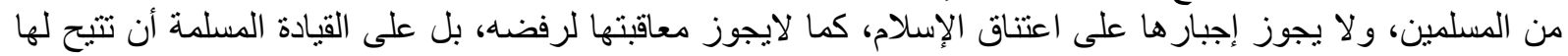
حرية الحركة، وتدخلها في حمايتها حتى تصل الصار آمنة إلى بلادها.

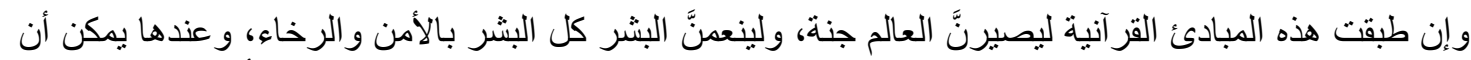



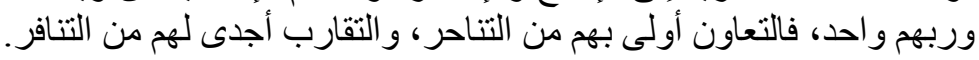

\section{ثبت المصادر والمراجع}

ابن أبي زَمَنِين، محمد بن عبد الله. (2002م). "تفسير القرآن العزيز" تحقيق: بن عكاثشة، حسين ، الكنز ،محمد بن مصطفى . الفاروق الحديثة : القاهرة ،مصر.



ابن عبد السلام، عز الدين عبد العزيز .( 1996م). "تفسير العز بن عبد السلام (تفسير القرآن)، دار ابن حزم، بيروت، بروبان



ابن عاثشور ، محمد الطاهر .(1997 م). ، "التحرير و التنوير" ، دار سحنون للنشر و التوزيع، تونس، تونس.

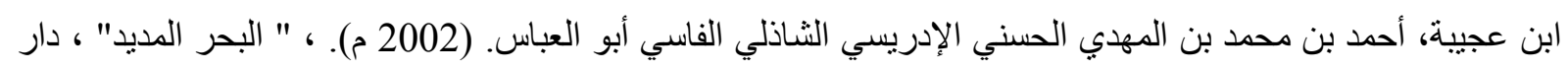
الكتب العلمية ـ بيروت، لبنان.

ابن كثير ، إسماعيل بن عمر القرشي الدمشقي. (1999م). " تفسير القرآن العظيم" تحقيق: سلامة، سامي بن محمد ـ دار طيبة للنشر و التوزيع: القاهرة ،مصر.

أبو السعود العمادي، محمد بن محمد .(بدون تاريخ). "إرشاد العقل السليم إلى مزايا الكتاب الكريم" دار الكتب العلمية، بيروت، لبنان. 
أبو الحسن، علي بن أحمد بن محمد بن علي الواحدي، النبسابوري، الثافعي. (1430 هـ). "التَفْْبيرُ البَبِيْطِ" عمادة البحث العلمي - جامعة الإمام محمد بن سعود الإسلامية.المدينة المنورة السعودية.

السمين الحلبي ، أحمد بن يوسف بن عبد الدائم. (المتوفى: 756هـ). "الدر الدصون في علوم الكتاب المكنون" تحقيق: الدكتور أحمد محمد الخر اط. دار القلم، بيروت، لبنان.

أبو حيان، محمد بن يوسف الأندلسي.(1420 هـ). "تفسير البحر المحيط" تحقيق : جميل، صدقي محمد. دار الفكر ، بيروت، لبنان بيروت.

أبو زهرة، محمد بن أحمد بن مصطفى.(1980م). "زهرة التفاسير "، دار الفكر العربي، القاهرة، مصر.

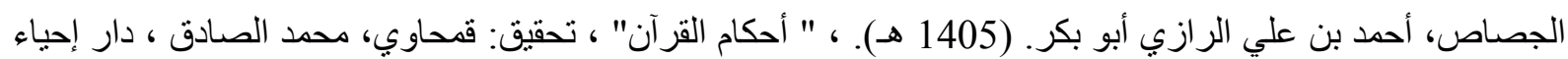

$$
\text { التزراث العربي - بيروت، لبنان. }
$$

البرصوي، إسماعيل حقي بن مصطفى الحنفي الخلوتي.( 2002 م) . ، " تفسير روح البيان" ، دار إحباء التراث العربى، القاهرة، مصر.

البغوي، محيي السنة، أبو محمد الحسين بن مسعود.( 1997 م). ، " معالم التنزيل" ، تحقيق: النمر، محمد عبد الهه ،


الثعلبي، أبو إسحاق أحمد بن محمد إبر اهيم.( 2005 م). ، "الكثف و البيان في تفسير القرآن" ، دار الكتب العلمية، بيروت، لبنان. الخازن، علاء الدين علي بن محمد بن إبراهيم بن عمر الثيحي أبو الحسن. (1415 هـ). ، " لباب التأويل في معاني التنزيل" ، دار الكتب العلمية ، بيروت ، لبنان. الجز ائري، جابر بن موسى بن عبد القادر بن جابر أبو بكر .( 2003م). ، "أيسر التفاسير لكلام العلي الكبير"، مكتبة العلوم و الحكم، المدينة المنورة، المملكة العربية السعودية. الزمخشري، أبو القاسم محمود بن عمر الخوارزمي. (1407 ه). ، "الكثاف عن حقائق التنزيل وعيون الأقاويل في وجوه التأويل" ، تحقيق : المهدي، عبد الرزاق ، دار إحياء التراث العربي ، بيروت ، لبنان.


اللويحق، مؤسسة الرسالة ، بيروت، لبنان.

الشعر اوي، محمد منولي. (1997 م). ، "تفسير الثعر اوي (الخواطر)"، مطابع أخبار اليوم، القاهرة، مصر.

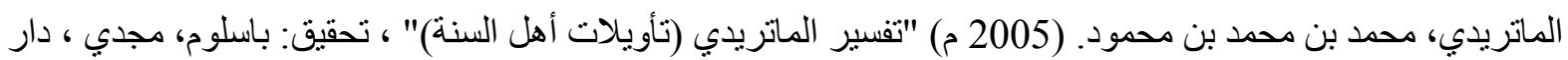
الكتب العلمية، بيروت، لبنان. الزحيلي، و هبة بن مصطفى.( 1422 هـ). ، "التفسير الوسيط "، دار الفكر - دمشق، سوريا. الصابوني، محمد علي. (1993م). "صفوة التفاسير"، دار التراث العربي، القاهرة، مصر. الطبري، محمد بن جرير ـ (2000).، "جامع البيان في تأويل القرآن" تحقيق: شاكر، أحمد محمد ، مؤسسة الرسالة، بيروت، لبنان.

العاني، عبد القادر بن ملّ حويش السيد محمود آل غازي. (1965 م). ، "بيان المعاني"، مطبعة الترقي، دمثق، سوريا. 
القرطبي، أبو عبد الله محمد بن أحمد بن أبي بكر بن فرح الأنصاري الخزرجي شمس الدين. (1964 م). ، " الجامع لأحكام

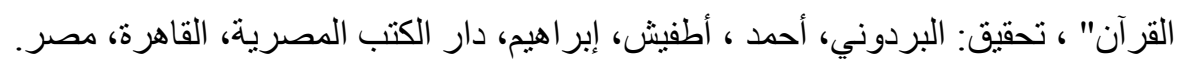

القثيري، عبد الكريم بن هوازن بن عبد الملك. (بدون). ، " لطائف الإشارات ( تفسير القثيري)" تحقيق: البسيوني،



الماوردي، أبو الحسن علي بن محمد بن محمد بن حبيب البصري البغدادي. (بدون). ، "تفسير الماوردي ( النكت

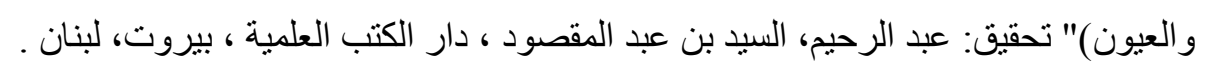

المظهري، محمد ثناء الله. (: 1412 هـ). "التفسير المظهري"، تحقيق: التونسي، غلام نبي ، مكتبة الرشدية ، باكستان.

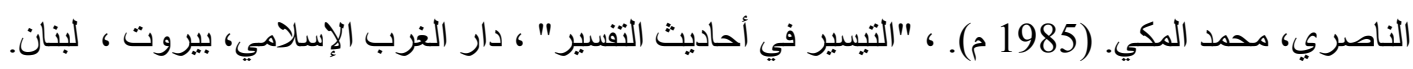
النسفي، أبو البركات عبد الله بن أحمد بن محمود(2005). " تفسير النسفى". تحقيق: الثعار ، مروان محمد دار النفائس بيروت

خلاف، عبد الو هاب ، علم أصول الفقه ،(1956) ـالطبعة السابعة ، مكتبة الدعوة الإسلامية شباب الأزهر، القاهرة مصر.

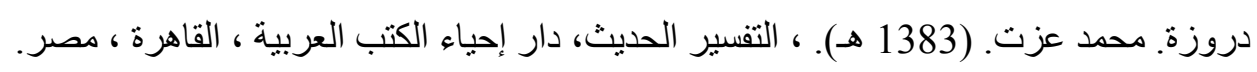
عبد الكريم يونس الخطيب، التفسير القرآني للقرآن، دار الفكر العربي ـ القاهرة، الطبعة الأولى 1383 ، 1970م .



$$
\text { قطب، سيد. (1987 م). ، في ظلال القرآن، دار الثروق، بيروت، لبنان. }
$$

Gündüzöz, Soner- Gündüzöz, Güldane.(2007)"Abdülhamid el-Kâtib, Doğunun Hükümdar1, çev." Antik Kitap, İstanbul, Türkiye.

\section{KAYNAKÇA}

Alusi, Ebu'1 Fadl. "Ruhu'1 Meani fi Tefsiri'1 Kur'an 1'1 Azim" , Beyrut, Lübnan.

Bağavi Ebu Ahmet, (1420)."Mealim u’t Tenzil ", 1.Bask1, thk . El-Mehdi, Abdurrazık , Beyrut , Lübnan.

Beyhaki Ebu Bekr, Sunen e'l Beyhaki e'l Kübra thk.M.Abdukadir Ata , Mekke,1414

Bursawi İsmail Hkkı bin Mustfa El-Hanafi(2002). " Tefsire'Rühü-L-Beyan ", Daru İhyai-t-turas ELArabi , Kahire, MISIR.

Cassas, Ahmed bin Ali Elrazi Ebübekir. (1405). " Ahkamü-L- Kur’an", thk. Kamhawiy, Muhammad El- Sadik, Daru İhyai-t-turas EL-Arabi, Beyrut, Lübnan.

Cezairiy,Cabir bin Musa bin Abdulkadir Ebü Bekir( 2003) " Eysrü-T- Tefasir likelami-L- Aliy Elkebir "Mektebetü-L- Ulum ve Elhikem Medine Sauudi Arabistan. 
Dürüze, Muhammed İzzet ( 1383) " Eltefsirü-L- Hdis", Daru İhyai-L-kütüp EL-Arabiye , Kahire , MISIR.

Ebu EL-Hasan EL-Nisaburiy, Ahmed bin Muhammed bin Ali EL-Wahidiy. (1430) ." EL-Tefsiri'l ELBesit " İmadetü-1-Bhsi EL-İlmiy,İmam Suud Ün.Medine, SUUDİ ARABİSTAN.

Ebu El-Suud El-Emadi, Muhammed bin Muhammed. (t.s). "İrşadi'l Akl es'Selim ile’l Mezeye e'l Kur'an i'1 Kerim", Tahkiksiz, Daru-l-Kuttab al-ilmiye, Beyrut, Lübnan.

Ebu Hayan, Muhammed bin Yusuf El-Endelusiy(1420) "Tefsiri'l EL-Behri-l-Muhit" Tahkik, Cemil Sidk1 Muhammed, Daru-l-Fikr, Beyrut, Lübnan.

Ebu Zehra Muhammed, (1980) " ZEHRATU-T-TAFASİR" , Daru-1-Fikr EL-Arabi, Kahire, MISIR.

Elaniy,Abdulkadir Mulla huwaiş Elsseyid Mahmud(2002). " Beyanü-L- Maaniy", Mattbatu-TTarakkiy, Dimaşk Süriye.

El-Halabi,Ahmed bin Yusuf bin Abdu-Daim. " Eldürü-l-Masun fi ulumi-1-Kitabi Elmeknun" Tahkik,Ahmed Elharrat Daru-1-Kalem, Beyrut, Lübnan.

Gündüzöz, Soner- Gündüzöz, Güldane.(2007). "Abdülhamid el-Kâtib, Doğunun Hükümdarı, çev." Antik Kitap, İstanbul, Türkiye.

Hallaf, Abdulwahab, .(1956) "İlmu Usulu-L- Fıkıh" ,7.Baskı Mektebetü- D- Dava Elislamiye, Kahire, MISIR.

Hatıp, Abdulkerim yunus.(1970) "Eltefsirü-L- Kur'aniy lilKur’an" , Daru-l-Fikr EL-Arabi, Kahire, MISIR.

Hazin, Alaauttin Ali bin Muhammed.(1415), "Lübabü-t-Tevil fi maany-t-Tenzil" Daru-l-Kuttab alilmiye, Beyrut, Lübnan.

İbn Abi Zamanin, Muhammed bin Abdullah. (2002). "Tefsiri'l Kur'an 1'l Aziz" , Tahkik, Bin Okasha,Hüseyin,Elkiniz, Muhammed Mustafa,Elfaruk Elhadise yaının evi, Kahire, MISIR.

İbnü Abdissalam,İzzüttin Abdulaziz. (1996 ). " Tefsiri’lIZZZ İBNİ Abdissalam" . Daru İBNİ Hazm, Beyrut, Lübnan.

İbnü Acibah,Ahmed bin Muhammed El-Mehdi El- Hüseyini(2002). "El-Bahrü El-Medid", Daru-1Kuttab al-ilmiye, Beyrut, Lübnan.

İbnü Aşur, Muhammed Eltahir. ( 1997) . " Eltehrir ve Eltenvir", Daru Sahnun, Tunus, Tunus.

İbnü El-Arabi,(1946). " Fususu-1-hikem" . Daru-1-Kitap El- ARABİ, Beyrut, Lübnan.

İbnü Kesir, Ebu'l Fide. (1999). "Tefsiri'l Kur'ani'l Azim" . Tahkik,Salamah, Sami bin Muhammed,Darü-Tıba, Kahire , MISIR.IZMIRLİ İ.Hakkı Kur’an-I Kerim ve Türkçe Tercümesi,İstanbul,1926

Kuran-1 Kerim Meali,Hazırlayanlar,Ziya Kazıcı , Necip Taylan , İstanbul , 1993

Kurtubi „Ebu'l Abdulah. (1964). " Tefsir'l Kurtubi", thk.Berduni Ahmet , Daru-1-Kütübi-L-Misriye, Kahire, MISIR.

Kuşeyri, Abdulkerim bin Hawzin bin Abdilmelik,( Tarihsiz) Lataifü-L- İşarat thk.Elbesyüni, İbrahim, Elheyaetü-L-Misryetü Elamme lilkitap, Kahire, MISIR.

Kutub ,Seyyid. (1987). " Fizilal E’l Kur’an" , Daru-Ş-Şuruk, Beyrut, Lübnan.

Matüridiy, Muhammed bin Muhammed bin Mahmud, (2005) " Tefsiri Matüridiy ". Daru-1-Kütüb Elilmiye, Beyrut, Lübnan. 
Mavirdiy,Eü Elhasan Ali bin Muhammed( tarihsiz) "Elnüket ve Eluyun" thk.Abdurrahim, Elseyit bin Abdilmaksud, Daru-1- Kütüb El-ilmiye, Beyrut, Lübnan.

Mazhariy,Muhammed senaü-l-lah.(1412) "Eltefsir Elmazhariy", thk.Eltünüsiy, Ğulam nebiy, Mektebet-R-ruşdiye,Pakistan.

Nahhas Ebu Cafer, " Meani El Kur'an El Kerim" , thk.M.Ali e’s Sabuni , 1.Bask1, Mekke, 1409

Nasıriy, Muhammed Elmekkiy.( 1985). " Elteysirü fi Ahadisi Eltefsir " , Daru-L-ğarbi Elislamiy, Beyrut, Lübnan.

Nesefi,Eb Elbaraket Ahmed bin Mahmud, (2005) " Tefsiri Nesefi". tehkik, Elşaar, Marawan Muhammed Daru-L-nefais, Beyrut, Lübnan.

Raşid Rıza, Muhammed bin Ali,(1990) " Tefsirü-L- Kur'ani Elhakim", Elheyaetü-L-Mısrıyetü Elamme lilkitap, Kahire, MISIR.

Saa'libi,Ebü İshak Ahmed bin Muhammed bin İbrahim.(2005). " El-keşfü ve El-beyan fi Tefsir-i Kur'an" , Tahkiksiz , Daru-1-Kuttab al-ilmiye, Beyrut, Lübnan.

Sabuni, Muhammed Ali. ( 1993)."Safvetu’t Tefasir", Daru Elturas ELArabi , Kahire , MISIR.

Sadiy, Abdurrahman bin Nsır ( 1423) " teysirü -L- Kerim Elrahman fi Tefsir-i Kelamü-L- Mennan" , Tahkik, Abdurrahman bin Mualla Elluwihık, Müessesetü-R- Risale, Beyrut, Lübnan.

Şarawiy, Muhammed Mutevelli ,(1997) . "Tefsire'-Ş- Şarawiy" Mtabiu Ahbar Elyevm, Kahire , MISIR.

Tabari, Muhammed bin Cerir. ( 2000). " Tefsire’t Tabari",Tahkik, Şakir, Ahmed Muhammed, Müessestü Elrisale, Beyrut, Lübnan.

Zamahşeri, Ebü-1-Kasim Mahmud bin Umran El Hawarizmiy.(1407) Elkeşşaf An hakaiki-t-Tenzil ve uyunu-L- Akawil fi vüvühü-T-Tevil, Daru İhyai-t-turas EL-Arabi , Beyrut, Lübnan.

Zühayli, Wahbah bin Mustafa.( 1422). Daru Elfikr, Dimeşk, Suriye. 